\title{
MAPA DE RISCO NO BRASIL: ELABORAÇÃO E IMPLANTAÇÃO EM UMA UNIDADE DE SERVIÇO \\ DE HEMATOLOGIA/ONCOLOGIA E TRANSPLANTE DE MEDULA ÓSSEA EM UMHOSPITAL UNIVERSITÁRIO
}

\author{
Planning and Implementing a Risk Map in a Department of Hematology/Oncology and Bone \\ Marrow Transplantation in a School Hospital in Brazil
}

Rosmari Hornerl, Marinês Calegari Lavall', Everton Boff, Iria Luiza Gomes Farias², Mariane de Mello Maraschin²

\begin{abstract}
RESUMO
Neste estudo relatamos as limitações encontradas na aplicação do Mapa de Risco no Brasil, sob o ponto de vista legal. Inicialmente são apresentadas a conceituação, origem e importância do mapa de risco. A seguir, os autores elaboraram um questionário, em que os trabalhadores envolvidos descreviam os riscos que identificavam na unidade de trabalho que estavam trabalhando. Como conclusão comprovou-se no setor avaliado a existência de riscos biológicos, físicos, químicos, ergonômicos e de acidentes de trabalho. Finalmente, implantou-se o mapa de risco no Serviço de Hematologia/oncologia e Transplante de Medula Óssea do Hospital Universitário de Santa Maria (HUSM).

Palavras-chave: Mapa de risco; Saúde do trabalhador; Unidade de hematologia/oncologia.

SUMMARY

Here we report the limitations encountered in applying a Risk Map in Brazil, under the legal point of view. First we describe the concept, origin and significance of the risk map. Next we develop a questionnaire in which the workers could report the risks they identified in their workplace. In conclusion, biological, physical, chemical, and ergonomic risks as well as risk of workplace accidents were proved to exist in the evaluated department. Finally, the risk map was implemented in the Department of Hematology/Oncology and Bone Marrow Transplantation in the University Hospital of Santa Maria (HUSM).

Keywords: Risk map, worker's health, department of hematology/oncology
\end{abstract}

\section{INTRODUÇÃO}

Por definição “a biossegurança é o conjunto de ações voltadas para a prevenção, minimização ou eliminação de riscos inerentes às atividades de pesquisa, produção, ensino, desenvolvimento tecnológico e prestação de serviços, riscos que podem comprometer a saúde do homem, dos animais, do meio ambiente ou a qualidade dos trabalhos desenvolvidos" ". Apesar do trabalho na área da saúde ter como característica básica a presença iminente de riscos biológicos, químicos, físicos, ergonômicos, ambientais e acidentes em sua atividade diária, a biossegurança não se restringe aos ambientes de saúde. Hoje existe uma tendência de mudança cultural, em nível mundial, com relação à obtenção de novos padrões de comportamento direcionado à preservação do meio ambiente e da própria vida. É imprescindível que todos os profissionais dos mais diversos ramos estejam conscientes dos riscos aos quais estão expostos para assim adotarem procedimentos de segurança na sua rotina

Trabalho desenvolvido na unidade de Serviço de Hematologia/oncologia e Transplante de Medula Óssea em um Hospital Universitário de Santa Maria (HUSM), sob a orientação do Programa de Pós-Graduação em Ciências Farmacêuticas

'Docente do Departamento de Análises Clínicas e Toxicológicas e do - Programa de Pós-Graduação em Ciências Farmacêuticas do Centro de Ciências da Saúde (CCS) da UFSM

Alunos do Programa de Pós-Graduação em Ciências Farmacêuticas do CCS/UFSM à nível de mestrado 
de trabalho ${ }^{4}$. A noção de risco tem a ver com a possibilidade de perda ou dano, ou como sinônimo de perigo. Neste estudo adotaremos uma concepção abrangente de risco e que se relaciona à saúde dos trabalhadores, significando toda e qualquer possibilidade de que algum elemento ou circunstância existente num dado processo e ambiente de trabalho possa causar dano à saúde, seja através de acidentes, doenças, ou ainda através da poluição ambiental. É claro que a saúde dos trabalhadores é muito mais abrangente do que os riscos nos locais de trabalho, e tem a ver com as condições mais gerais de trabalho e vida, como salário, moradia, alimentação, lazer, existência de creche no trabalho, participação nas decisões da sociedade, entre outras. O próprio trabalho, quando realizado num ambiente saudável, pode ser uma importante fonte de saúde. As atividades capazes de proporcionar danos, doença ou morte para os seres vivos são caracterizadas como atividades de risco. "Risco é a probabilidade de ocorrer um evento bem definido no espaço e no tempo, que cause dano à saúde, às unidades operacionais ou dano econômico/financeiro. $\mathrm{Na}$ presença de um perigo, não existe risco zero, porém existe a possibilidade de minimizá-lo ou alterá-lo para níveis considerados aceitáveis"

Historicamente, o conceito de risco nos locais de trabalho foi inicialmente concebido como os riscos ocupacionais clássicos que geram conseqüências mais diretas e visíveis, gerando os acidentes de trabalho $\mathrm{e}$ as doenças diretamente relacionadas ao trabalho. Posteriormente, com a luta dos trabalhadores e os avanços de campos como a ergonomia e as ciências de risco, outros conceitos foram desenvolvidos. Dessa forma, os riscos saíram do campo das conseqüências do ambiente físico, das máquinas, equipamentos, produtos e substâncias, mas estão inseridos com a organização do trabalho e as formas de gerenciamento. São considerados pertencentes a uma população de risco os trabalhadores que prestam assistência a pacientes, incluindo técnicos e profissionais de laboratórios, estudantes da área de saúde, enfim todos aqueles que dão apoio à prestação de serviços médicos. Sendo assim, a prevenção por meio de equipamentos de proteção individual (EPIs) e coletiva (EPCs) e educação com a formação de uma consciência de prevenção, são fundamentais ${ }^{8}$.

Existem metodologias para o levantamento de riscos, as quais podem ser caracterizadas em dois grupos: as retrospectivas que se baseiam em antecedentes, ou seja, fatos já ocorridos e as prospectivas, as quais possuem um caráter exploratório, que permitem a antecipação, a correção de falhas antes de se manifestarem concretamente. Os métodos prospectivos que mais se destacam são: o método LEST e o mapa de risco (MR). $O$ mapa de risco tem sido a metodologia mais utilizada no mundo, e teve sua origem no modelo operário italiano (MOI) ${ }^{4}$, não sendo, portanto desenvolvido nos meios acadêmicos. O MR é uma representação gráfica de um conjunto de fatores presentes nos locais de trabalho, capazes de acarretar prejuízos à saúde do trabalhador. Tais fatores se originam nos diversos elementos do processo de trabalho (materiais, equipamentos, instalações, suprimentos, e nos espaços de trabalho, onde ocorrem as transformações) e da forma de organização do trabalho, (arranjo físico, ritmo de trabalho, método de trabalho, turnos de trabalho, treinamento, etc $)^{3}$. A realização do mapeamento de riscos tornou-se obrigatória no Brasil para todas as empresas que tenham a CIPA (Comissão Interna de Prevenção de Acidentes), através da Portaria número 5 de 17/08/92 do Departamento de Segurança a Saúde do Trabalhador do Ministério do Trabalho. A CIPA, para a realização do MR poderá contar com a colaboração do serviço especializado em Engenharia de Segurança e em Medicina do Trabalho (SESMT) da empresa, caso exista ${ }^{2}$.

A classificação de riscos no ambiente de trabalho é definida pela portaria 3.214/78 do Ministério do Trabalho e Normas Regulatórias de Medicina e Segurança do Trabalho ${ }^{1,5}$. São cinco os principais riscos:

- Risco físico- NR09 e NR15 - formas de energia a que possam estar expostos trabalhadores. A caracterização dos riscos físicos é feita através de avaliações ambientais quantitativas;

- Riscos químicos- NR09 e NR15- substâncias, compostos ou produtos que possam penetrar no organismo pela via respiratória, nas formas de poeira, fumos, névoas, neblinas, gases ou vapores, ou que pela natureza da atividade de exposição, possam ter contato com a pele ou ser absorvidos pelo organismo através da pele ou por ingestão A caracterização dos riscos físicos é feita através de avaliações ambientais quantitativas e qualitativas;

- Riscos biológicos- NR09- bactérias, bacilos, parasitas, protozoários, vírus entre outros. Riscos ergonômicos-NR17elementos físicos e organizacionais que interferem no conforto da atividade laboral e, consequentemente, nas características psicofisiológicas do trabalhador;

- Riscos de acidentes - arranjo físico, eletricidade, máquinas e equipamentos, incêndio/explosão, etc ${ }^{1,5}$.

Com relação ao mapa de risco, seu produto final visual costuma ser uma planta baixa ou croqui do local de trabalho, com círculos coloridos que representam os riscos encontrados. Os mapas devem ser fixados em locais visíveis em todas as seções da empresa, para que os trabalhadores possam visualizá-los. Porém, mais importante que o resultado gráfico do MR, constitui-se o processo de participação e envolvimento dos trabalhadores na sua construção. Este é o ponto importante e que queremos ressaltar.

A elaboração de mapa de risco é um processo educativo e organizativo que pode abrir espaço para que as pessoas envolvidas reflitam sobre o seu próprio trabalho e aprendam sobre o trabalho dos colegas ${ }^{4,6}$.

O objetivo do nosso trabalho foi à elaboração do mapa de risco no setor de Hematologia/Oncologia do HUSM - Santa Maria, RS, fornecendo conceitos e métodos de compreensão, bem como estratégias de intervenção e controle dos riscos 
neste local de trabalho, melhorando as condições de trabalho.

\section{MATERIAL E MÉTODOS}

O setor de Hematologia/Oncologia do Hospital Universitário de Santa Maria (HUSM) está localizado no andar térreo e possui uma área física de aproximadamente $215 \mathrm{~m}^{2}$. Este setor é subdividido em onze subsetores constituídos pela secretaria, coleta, rotina, setor de genética, laboratório de histocompatibilidade (HLA), biologia molecular, criopreservação, citometria de fluxo, microscopia, sala de armazenamento do material biológico (sala dos freezers) e setor da limpeza. Além dos sanitários, sala de reuniões e vestuário, no qual trinta e um funcionários atuam desempenhando as funções de farmacêuticos, médicos, profissionais especialistas em biologia molecular, auxiliares de limpeza e administrativos, além dos alunos de graduação da UFSM que compõem o quadro de bolsistas.

O estudo foi realizado no período de março a outubro de 2005 e consistiu de 3 encontros com os profissionais deste laboratório. No primeiro encontro, foi realizada uma entrevista semi-estruturada com questões referentes ao conhecimento prévio da legislação de biossegurança e mapa de risco. Após, foram levantados os riscos de cada subsetor, de acordo com as atividades realizadas. O dimensionamento do risco (pequeno, médio, grande) foi estabelecido em consenso pelos funcionários, baseado apenas em suas percepções, não utilizando nenhum método quantitativo. No segundo momento, com os funcionários reunidos em grupos, foi preenchido um formulário por setor, de acordo com o modelo proposto por Ponzetto ${ }^{6}$ e elaborado o mapa de risco propriamente dito. A análise dos dados coletados foi realizada manualmente pelos estudantes do curso de Pós-Graduação em Ciências Farmacêuticas ao nível de mestrado, da UFSM, autores deste estudo, supervisionados pela docente da disciplina Tópicos Avançados em Bioanálises. De posse desses dados foi realizado o terceiro encontro com os funcionários, os pesquisadores e um técnico em segurança do trabalho, para avaliação e discussão.

Os trabalhadores participaram de forma voluntária, sendo que suas identidades e respostas foram preservadas. No primeiro encontro foi assegurado o retorno dos resultados da pesquisa e o compromisso da elaboração do mapa de risco.

\section{RESULTADOSE DISCUSSÃO}

O formulário aplicado aos funcionários continha questões referentes ao tipo de atividade realizada em cada subsetor, número de funcionários, tipos de equipamentos de proteção individual e coletiva utilizados rotineiramente em seus serviços, riscos de acidentes que temiam estarem expostos no desempenhar de suas funções e citação dos acidentes que haviam ocorrido nos últimos doze meses.

Os tipos de riscos e sua presença em determinado subsetor podem ser visualizados na Tabela 1 , na qual nos chamam atenção os riscos ergonômicos, que estão presentes em $100 \%$ dos subsetores e apresentam natureza variada. As tarefas de repetição como pipetagem e contagem de células geraram duas ocorrências de tendinite nos últimos doze meses. Para os riscos ergonômicos neste setor nenhuma medida preventiva foi instituída uma vez que este tipo de risco está diretamente relacionado aos elementos do processo de trabalho, como a jornada prolongada, imposição de uma rotina intensa, entre outros fatores.

A exposição ao risco biológico está presente em $90,9 \%$ dos subsetores, excluindo-se apenas na secretaria. Os funcionários encontram-se constantemente expostos a materiais biológicos nobres e potencialmente infectantes (sangue, líquor e medula óssea). Embora o uso de aventais e luvas minimize os riscos de contaminação frente ao derramamento de fluidos, mas não previne os acidentes do tipo perfuro cortante, os quais devem ser evitados pelo próprio manipulador através do conhecimento e da utilização das boas práticas de biossegurança constantes nas NR06 (equipamento de proteção individual) e NR09 (programa de prevenção de riscos ambientais). Apesar de todas as medidas protetivas em vigor, dois acidentes com agulhas foram registrados durante $o$ período em questão ${ }^{5}$.

O risco físico está presente em $36,36 \%$ dos subsetores e nos últimos doze meses não houve relato de acidentes. Para a exposição a esta classe de risco, luvas e protetores auriculares são as medidas protetivas utilizadas pelo setor.

A manipulação de reagentes químicos potencialmente tóxicos, cancerígenos ou inflamáveis está presente em 45,45\% dos sub-setores, onde as luvas e aventais são os principais EPIs e a utilização de capela de exaustão (EPC) protege os funcionários do risco de inalar substâncias voláteis. Entretanto aponta-se a falta de um local adequado para o armazenamento de alguns reagentes. Nos últimos doze meses apenas um episódio de contato com reagentes químicos no rosto e nas mãos foi descrito.

Como conseqüência das atividades realizadas no setor de Hematologia/Oncologia o risco de acidente está presente em $90,9 \%$ dos subsetores, excetuando-se novamente a secretaria. Nos últimos doze meses além dos acidentes já citados, tem-se o registro de uma queimadura em um funcionário da limpeza durante a manipulação do ebulidor. 
Tabela 1 - Distribuição dos tipos de riscos e presença nos subsetores apontados pelos trabalhadores da unidade de Serviço de Hematologia/oncologia e Transplante de Medula Óssea do HUSM

\begin{tabular}{|c|c|c|c|c|}
\hline Tipo de Risco & $\mathbf{N}$ & $\%$ & Riscos Apontados & Setores \\
\hline \multirow{10}{*}{ ERGONÔMICO } & \multirow{10}{*}{11} & \multirow{10}{*}{100} & Mobiliário inadequado & $\mathrm{BM}, \mathrm{CF}, \mathrm{G}, \mathrm{H}, \mathrm{M}$ \\
\hline & & & Trabalho em pé & G, L \\
\hline & & & Trabalho repetitivo (pipetagem, contagem). & $\mathrm{CF}, \mathrm{H}, \mathrm{M}$ \\
\hline & & & barulho & $\mathrm{B}, \mathrm{C}, \mathrm{CP}, \mathrm{F}, \mathrm{H}, \mathrm{M}, \mathrm{R}, \mathrm{S}$ \\
\hline & & & Stress, psicológico & $\mathrm{C}, \mathrm{R}, \mathrm{S}$ \\
\hline & & & Ventilação/iluminação & $\mathrm{BM}, \mathrm{C}, \mathrm{CF}, \mathrm{CP}, \mathrm{F}, \mathrm{G}, \mathrm{L}, \mathrm{M}, \mathrm{R}, \mathrm{S}$ \\
\hline & & & Levantamento peso & $\mathrm{CP}$ \\
\hline & & & Atividade tempo prolongado contínuo & $\mathrm{CF}, \mathrm{H}, \mathrm{S}$ \\
\hline & & & Espaço físico pequeno em relação $\mathrm{n}^{\circ} \mathrm{de}$ & $\mathrm{BM}, \mathrm{C}, \mathrm{M}, \mathrm{R}$ \\
\hline & & & pessoas trabalhando & \\
\hline \multirow{4}{*}{ BIOLÓGICO } & \multirow{3}{*}{10} & \multirow{3}{*}{90,9} & Manipulação material biológico & $\mathrm{BM}, \mathrm{C}, \mathrm{CF}, \mathrm{CP}, \mathrm{F}, \mathrm{G}, \mathrm{H}, \mathrm{L}, \mathrm{M}, \mathrm{R}$ \\
\hline & & & Transporte material biológico e deslocamento & $\mathrm{CF} \mathrm{H}$ \\
\hline & & & em áreas não contínuas & \\
\hline & \multirow{3}{*}{5} & \multirow{3}{*}{45,45} & Freezer- $80^{\circ} \mathrm{C}$ & F H \\
\hline \multirow[t]{2}{*}{ FÍSICO } & & & Ruído intenso & $\mathrm{CP}$ \\
\hline & & & Nitrogênio líquido & $\mathrm{CP}$ \\
\hline \multirow[t]{2}{*}{ QUÍMICO } & 5 & 45,45 & Reativos tóxicos & BM CP G L R \\
\hline & \multirow{3}{*}{10} & \multirow{3}{*}{90,9} & Espaço para circulação inadequado & BM C G L M R \\
\hline \multirow[t]{2}{*}{ ACIDENTE } & & & Perfuro cortante & BM C CP G H L \\
\hline & & & Selador, ebulidor & CP L \\
\hline
\end{tabular}

BM: biologia molecular; C: coleta; CF: citometria de fluxo; CP: crio preservação; F: sala de freezer; G: genética; H: histocompatibilidade; L: limpeza; M: microscopia; R: rotina; S: secretaria;

O trabalho culminou com a elaboração do Mapa de Risco da Unidade, em conjunto com os trabalhadores que responderam o questionário, e está representado na Figura 1. 
Figura 1: Mapa de risco da unidade de Serviço de Hematologia/oncologia e Transplante de Medula Óssea do HUSM elaborado com a participação dos trabalhadores do setor, Santa Maria, 2005.
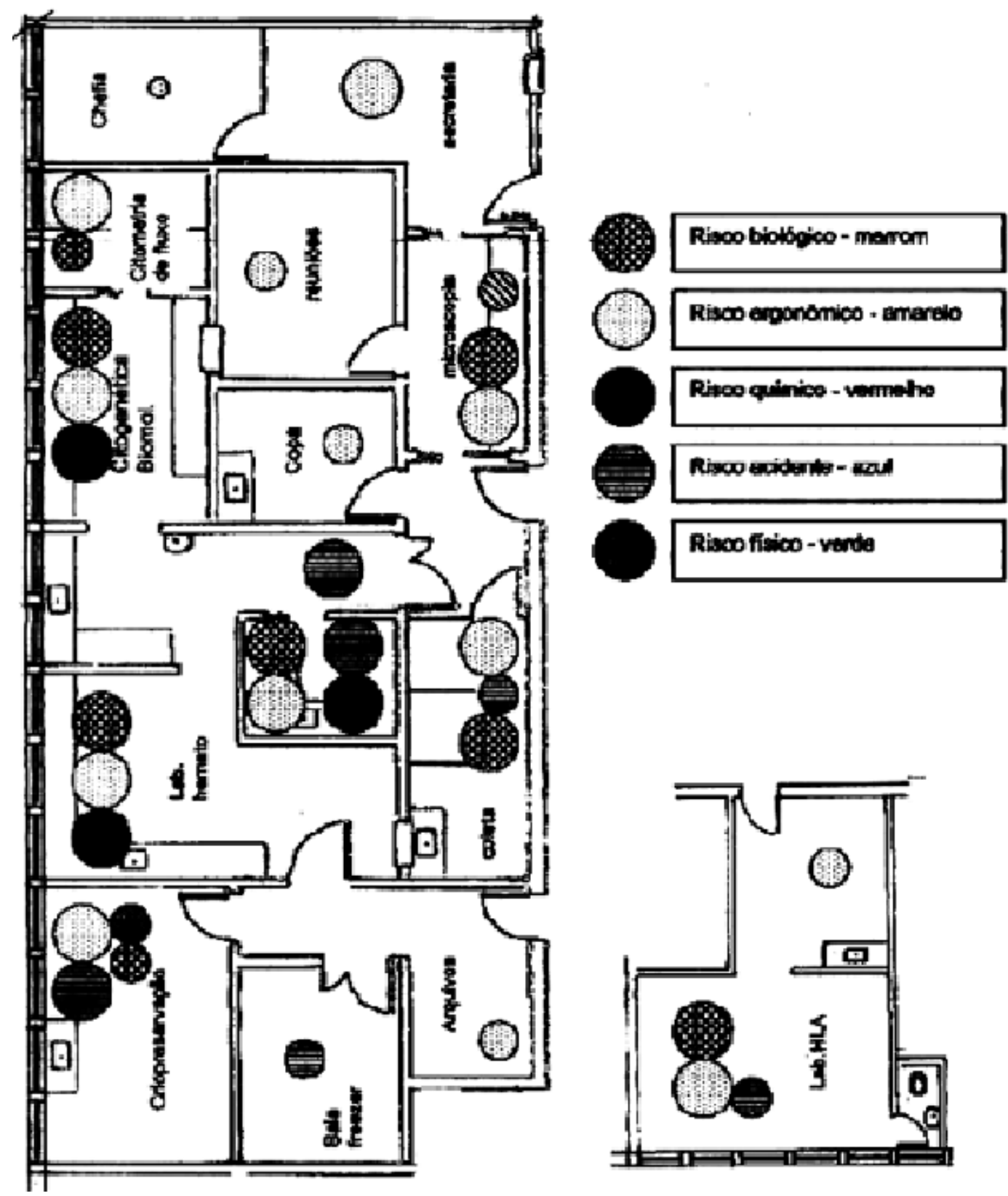

\section{CONCLUSÃO}

O modelo proposto por Ponzetto para elaboração de mapa de risco mostrou-se uma ferramenta fundamental para que $o$ grupo conseguisse elaborar o mapa de risco. Todos os funcionários tinham clareza dos riscos aos quais estavam expostos, embora a maioria não tivesse conhecimento específico do que significava mapa de risco. As maiores dificuldades encontradas referiram-se a quantificação dos riscos. O modelo citado contém a informação de "como fazer". Acreditamos que todo e qualquer trabalhador que trabalha na área da saúde e, portanto com materiais biológicos, quer seja na prestação de serviços à população (hospitais, laboratórios, unidades básicas de saúde - UBS) ou de caráter acadêmico (ensino, pesquisa ou extensão) deve 
elaborar o mapa de risco de seu local de atuação, como exercício para prevenir acidentes, uma vez, que por definição, o mapa de risco é uma representação gráfica de um conjunto de fatores presentes nos locais de trabalho, capazes de acarretar prejuízos à saúde dos trabalhadores. O mapa de risco propicia uma maior conscientização de todas as pessoas que atuam num mesmo local de trabalho, não só dos riscos existentes, mas principalmente, de sua responsabilidade frente a estes riscos. É responsabilidade de todos não colocar em risco seus colegas, ambiente e a si próprio. O mapa de risco poderia ser visto não como mais uma exigência da legislação, sujeita à fiscalização, um "papel na parede", mas como um exercício coletivo que favorece o comprometimento das pessoas com a biossegurança.

\section{REFERÊNCIAS BIBLIOGRÁFICAS}

1. Teixeira P, Valle S, organizadores. Apresentação - Biossegurança - Uma abordagem multidisciplinar. Rio de Janeiro: Editora Fiocruz; $1998 ; 13-14$

Endereço para correspondência:

Rosmari Hörner

Departamento de Análises Clínicas e Toxicológicas - Centro de Ciências da Saúde - Universidade Federal de Santa Maria - Avenida Roraima $n^{0} 1000$ - Cidade Universitária - Prédio n ${ }^{0} 26$, segundo andar - Salas 1205-1201 - Bairro Camobi -

Santa Maria - RS - CEP: 97105-900 - Telefone: (55)-3220-8464 e-mail: rosmari@smail.ufsm.br
2. Mattos UAO, Freitas NBB. Mapa de risco no Brasil: as limitações da aplicabilidade de um modelo operário - Caderno de Saúde Pública 1994; 251-258

3. Xavier RM, Albuquerque GC, Barros E, organizadores. Laboratório na prática clínica - Consulta rápida. Porto Alegre: Editora Artmed; 2005; 118-122.

4. Mattos UAO, Santos PR, Mastroeni MF, organizadores. Biossegurança aplicada a Laboratórios e Serviços de Saúde. São Paulo: Editora Atheneu; 2004; 94-117.

5. CIPAS - Comissões Internas de Prevenção de Acidentes nas Empresas. http://www.btu.unesp.br/cipa/mapaderisco.htm (acessado em 10/Jun/2005).

6. Brasil-Ministério da Saúde. Saúde ambiental e gestão de resíduos de serviços de saúde. Brasília: MS, 2002. Série comunicação e educação em saúde-Projeto Refarsus, capacitação à distância. p. 82 .

7. Ministério do Trabalho e Emprego: Normas Regulamentadoras - NR. http://www.mtb.gov.br/Empregador/segsau/Legislacao/ Normas/Default.asp_(acessado em 30/Nov/2005).

8. Ponzetto G. Mapa de riscos ambientais: manual prático. Ed. LTR: São Paulo, 2002 